\title{
新西兰陶波酸性火山岩氧 同位素组成研究*
}

\author{
胡瑞忠 \\ (中国科学院地球化学研究所, 贵阳 550002) \\ P. Blattner \\ (新西兰地质与核科学研究所,下哈特)
}

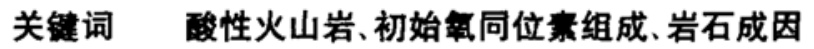

新西兰北岛陶波 (Taupo) 火山岩带, 位于太平洋板块俯冲于印度板块之下的印度板块一 侧, 是世界上最著名的第四纪酸性火山岩带之一, 长约 $250 \mathrm{~km}$, 宽约 $50 \mathrm{~km}$, 其中 $95 \%$ 以上的 岩石为 $\mathrm{SiO}_{2}$ 含量大于 $70 \mathrm{wt} . \%$ 、含石英和长石斑晶的凝灰岩和流纹岩 ${ }^{[1]}$. 该带形成于拉张构造 环境,空间上呈北东向与陶波地堑的展布相吻合 ${ }^{[2]}$.

由于水 - 岩作用的结果, 表面上看来很新鲜的火山岩, 其实测氧同位素组成亦可能大大偏 离初始值 ${ }^{[3,4]}$, 因而用全岩样品的实测值解释岩石成因将具有不确定性. 本文首次提供了陶波 火山岩带酸性火山岩中长石和石英斑晶的氧同位素数据; 根据对单矿物氧同位素的研究, 恢 复了全岩样品的初始氧同位素组成, 在此基础上探讨了岩石成因.

\section{1 火山岩的氧同位素组成}

\section{1 分析方法与结果}

本研究分别测定了火山岩全岩样品以及从中分离出的长石 (所有长石的混合物) 和石英斑 晶的 27 套氧同位素数据 (表 1), 分析工作在新西兰地质与核科学研究所完成. $\mathrm{CO}_{2}$ 气体样品的 制备采用 $\mathrm{BrF}_{5}$ 系统, 然后在 NAA6-60-RMS 质谱上测定其同位素含量. 数据处理时标准样 NES- 28 的 $\delta^{18} \mathrm{O}$ 值采用 $9.60 \%$, 对重复测过的样品, 表 1 给出了误差值, 平均误差约为 $0.16 \% 0$.

\section{2 长石和石英的筑同位素关系}

长石和石英与流体的氧同位素交换速度存在着很大差异 ${ }^{[9]}$. 因此, 石英与长石之间氧同 位素分馏实测值的大小, 可以指示它们在成岩后可能发生的变化. 如果长石和石英或者其中 之一成岩后与流体发生过强烈的氧同位素交换, 那么它们之间将会出现异常的氧同位素分馏 值. 对于陶波火山岩带中的酸性火山岩, 实际情况是: (1) 随机测定的 14 个样品, 长石与石英矿 物对的氧同位素测定值呈很好的正相关 (图 1), 用最小二乘法对其进行处理确定出:

1993-03-29 收稿, 1993-08-29 收修改稿.

*新西兰 DSIR 国际合作基金资助项目. 
表 1 陶波火山岩带酸性火山岩的氧同位素组成 ${ }^{\mathrm{a} ;}$

\begin{tabular}{|c|c|c|c|c|c|c|c|c|}
\hline 序号 & 样品号 & 岩性 & $\delta^{18} \mathrm{O}_{Q}$ & $\delta^{18} \mathrm{O}_{\mathrm{F}}$ & $\Delta_{Q-F}$ & $\delta^{18} \mathrm{O}_{\mathrm{TR}}$ & $\delta^{18} \mathrm{O}_{\mathrm{i}}$ & $\mathrm{SiO}_{2}$ (wt.\%) \\
\hline 1 & $14436 \mathrm{~A}$ & 流纹岩 & - & 6.86 & - & $7.58 \pm 0.10(4)$ & 7.20 & 75.67 \\
\hline 2 & $14436 \mathrm{~B}$ & 流纹岩 & - & 6.79 & - & $6.20 \pm 0.13(3)$ & 7.13 & 75.23 \\
\hline 3 & Whak2 & 凝灰岩 & $8.19 \pm 0.01(2)$ & $7.47 \pm 0.13(2)$ & 0.72 & $7.38 \pm 0.01(2)$ & 7.71 & 73.42 \\
\hline 4 & Whak3 & 凝灰岩 & $8.05 \pm 0.27(2)$ & 7.32 & 0.73 & $6.30 \pm 0.22(2)$ & 7.56 & 73.37 \\
\hline 5 & IG8098-2 & 数灰岩 & 8.17 & 7.29 & 0.88 & $7.50 \pm 0.23(2)$ & 7.58 & - \\
\hline 6 & WT52B & 凝灰岩 & - & $6.80 \pm 0.18(2)$ & - & 6.15 & 7.14 & - \\
\hline 7 & WT50D & 凝灰岩 & - & $7.14 \pm 0.05(2)$ & - & - & 7.45 & - \\
\hline 8 & WT50A & 准灰岩 & - & $7.52 \pm 0.21(2)$ & - & $8.15 \pm 0.05(2)$ & 7.80 & - \\
\hline 9 & WT25 & 凝灰岩 & 8.01 & $7.17 \pm 0.01(2)$ & 0.84 & - & 7.45 & - \\
\hline 10 & WT28 & 凝灰岩 & $8.02 \pm 0.15(3)$ & $7.05 \pm 0.15(2)$ & 0.97 & - & 7.37 & - \\
\hline 11 & WT34 & 颣灰岩 & 8.03 & - & - & - & 7.40 & - \\
\hline 12 & R 15126 & 凝灰岩 & - & 7.54 & - & $7.79 \pm 0.13(2)$ & 7.81 & 70.59 \\
\hline 13 & R 14586 & 凝灰岩 & 8.54 & - & - & $8.12 \pm 0.15(2)$ & 8.04 & 74.32 \\
\hline 14 & MM9(140m) & 岩灰 & $8.78 \pm 0.10(3)$ & - & - & $8.15 \pm 0.01(2)$ & 8.34 & - \\
\hline 15 & WK $302(218 \mathrm{~m})$ & 凝灰岩 & - & $8.07 \pm 0.24(2)$ & - & $8.92 \pm 0.02(2)$ & 8.29 & - \\
\hline 16 & $14395 \mathrm{~A}$ & 凝灰岩 & $7.67 \pm 0.23(2)$ & 6.60 & 1.07 & $7.75 \pm 0.09(2)$ & 6.96 & 72.34 \\
\hline 17 & $14397 \mathrm{~A}$ & 流纹岩 & - & 6.76 & - & $8.38 \pm 0.08(2)$ & 7.11 & 75.33 \\
\hline 18 & $14434 \mathrm{~A}$ & 凝灰岩 & - & 7.20 & - & $8.71 \pm 0.16(2)$ & 7.50 & 72.42 \\
\hline 19 & RH3 & 凝灰岩 & - & 8.02 & - & $9.10 \pm 0.18(2)$ & 8.25 & 74.41 \\
\hline 20 & $\mathrm{HH} 1$ & 凝灰岩 & $8.82 \pm 0.25(2)$ & 8.30 & 0.52 & - & 8.47 & 74.52 \\
\hline 21 & P30230 & 凝灰岩 & $8.08 \pm 0.20(2)$ & $6.96 \pm 0.15(2)$ & 1.12 & 7.58 & 7.33 & - \\
\hline 22 & $8086-10$ & 流纹岩 & $7.91 \pm 0.08(3)$ & 7.00 & 0.91 & - & 7.30 & 75.60 \\
\hline 23 & P39761 & 凝灰岩 & $7.74 \pm 0.20(3)$ & $6.71 \pm 0.08(2)$ & 1.03 & - & 7.05 & - \\
\hline 24 & $8068-12$ & 凝灰岩 & $8.54 \pm 0.12(3)$ & 7.90 & 0.64 & - & 8.11 & - \\
\hline 25 & P22957 & 岩灰 & $8.99 \pm 0.08(2)$ & $7.85 \pm 0.09(2)$ & 1.14 & - & 8.23 & - \\
\hline 26 & P22394 & 凝灰岩 & $7.57 \pm 0.18(4)$ & $6.38 \pm 0.01(2)$ & 1.19 & - & 6.78 & - \\
\hline 27 & P22395 & 凝灰岩 & $7.58 \pm 0.30(2)$ & 6.44 & 1.14 & - & 6.82 & - \\
\hline
\end{tabular}

a) 一表示未测项; 按 (2)式计算 $\delta^{18} \mathrm{O}_{\mathrm{i}}$ 时, 对本表中缺 $\delta^{18} \mathrm{O}_{\mathrm{Q}}$ 或 $\delta^{18} \mathrm{O}_{\mathrm{F}}$ 的样品,它们的值是按 (1)式的计算值; $\delta^{18} \mathrm{O}$ 值为相 对于 SMOW 的千分偏差, 各脚注的含义见正文; 括号中数字为测定次数.

$$
\delta^{18} \mathrm{O}_{\mathrm{Q}}=0.7233 \delta^{18} \mathrm{O}_{\mathrm{F}}+2.9065 \text {, }
$$

式中 $\delta^{18} \mathrm{O}_{\mathrm{Q}}$ 和 $\delta^{18} \mathrm{O}_{\mathrm{F}}$ 分别为石英和长石的氧 同位素实测值. 该方程的标准方差仅为 0.14 $\% 0$, 小于样品的平均分析误差 $0.16 \%$ ；（2） 14 个矿物对的 $\Delta_{\mathrm{Q}-\mathrm{F}}$ 值为 $0.52-1.19$ (表 1), 根据有关温度计确定的温度约为 $700-1100^{\circ} \mathrm{C}$. 这一 $\Delta$ 值和温度范围与世界 上酸性火成岩的正常范围相一致 ${ }^{[5,6]}$.

上述事实无疑表明: (1) 火山岩中的长石 和石英在结晶时与熔体达到了同位素平衡;

(2)该二矿物的实测氧同位素值基本可代表它

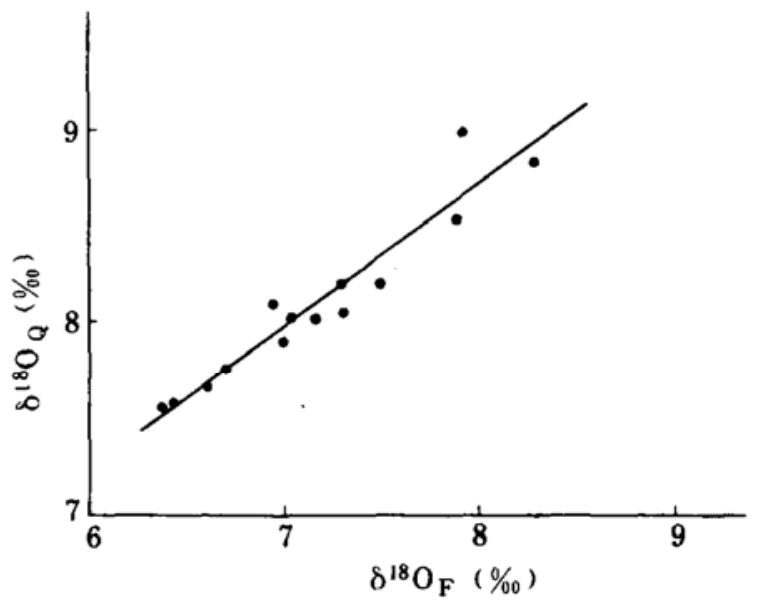

图 1 长石和石英斑晶的氧同位素组成关系 
们结晶时的初始值.

\section{3 全岩样品的氧同位素组成}

酸性火山岩 16 个全岩样品的 $\delta^{18} \mathrm{O}$ 实测值 $\left(\delta^{18} \mathrm{O}_{\mathrm{TR}}\right)$ 为 $6.20-9.10$ (表 1$)$, 同 $\delta^{18} \mathrm{O}_{\mathrm{F}}$ 与 $\delta^{18} \mathrm{O}_{\mathrm{Q}}$ 之间出现的良好线性关系 (图 1) 形成鲜明对比, $\delta^{18} \mathrm{O}_{\mathrm{TR}}$ 与 $\delta^{18} \mathrm{O}_{\mathrm{F}}$ 或 $\delta^{18} \mathrm{O}_{\mathrm{Q}}$ 之间几乎无任何相关 性 (图略). 相对于同一样品中的石英和长石, $\delta^{18} \mathrm{O}_{\mathrm{TR}}$ 存在三种情况: $\delta^{18} \mathrm{O}_{\mathrm{TR}}>\delta^{18} \mathrm{O}_{\mathrm{Q}}$, 如样品 $14395 \mathrm{~A} ; \delta^{18} \mathrm{O}_{\mathrm{F}}<\delta^{18} \mathrm{O}_{\mathrm{TR}}<\delta^{18} \mathrm{O}_{\mathrm{Q}}$, 如样品 IG8098-2 和 $\mathrm{P} 30230 ; \delta^{18} \mathrm{O}_{\mathrm{TR}}<\delta^{18} \mathrm{O}_{\mathrm{F}}$, 如样品 Whak2、 Whak3 和 WT52B.

初始火成岩中, 石英被公认为最富 ${ }^{18} \mathrm{O}$ 的造岩矿物. $\delta^{18} \mathrm{O}_{\mathrm{TR}}$ 的上述分布特征, 尤其是同一 样品中 $\delta^{18} \mathrm{O}_{\mathrm{TR}}>\delta^{18} \mathrm{O}_{\mathrm{Q}}$ 等现象的存在表明, 某些全岩样品的氧同位素组成在喷发成岩后发生了 明显变化. 如上所述, 样品中石英和长石的实测 $\delta^{18} \mathrm{O}$ 值基本可代表其初始特征, 因而我们有 理由认为, 部分全岩样品成岩后 $\delta^{18} \mathrm{O}$ 值的这些变化, 应主要由流体与火山岩中基质间的相互 作用所产生.

\section{4 火山岩的初始簕同位素组成}

以往恢复火山岩初始氧同位素组成主要采用两种方法：(1)根据全岩氧同位素实测值与全 岩水含量的关系外推 ${ }^{[4]} ;(2)$ 根据氧同位素组成未变化矿物的 $\delta^{18} \mathrm{O}$ 值及其结晶温度下与熔体间 的分馏系数计算 ${ }^{[3]}$. 本文中, 我们采用了另一种方法.

如上所述, 尽管某些陶波火山岩样品的基质部分在成岩后氧同位素组成变化明显, 但其中 长石和石英斑晶的实测 $\delta^{18} \mathrm{O}$ 值则基本保持了其初始特征. 化学成分的矿物标准化工作指 出, 陶波酸性火山岩各样品的标准矿物基本由一份石英和二份长石组成, 与石英和长石相比 其它标准矿物的含量常可忽略不计. 这些条件表明, 根据标准矿物成分对长石和石英的实 测 $\delta^{18} \mathrm{O}$ 值进行加权平均来恢复陶波酸性火山岩的初始 $\delta^{18} \mathrm{O}$ 值是可行的. 具体计算时, 我们 采用了以下公式:

$$
\delta^{18} \mathrm{O}_{\mathrm{i}}=\frac{1}{3} \delta^{18} \mathrm{O}_{\mathrm{Q}}+\frac{2}{3} \delta^{18} \mathrm{O}_{\mathrm{F}},
$$

式中 $\delta^{18} \mathrm{O}_{\mathrm{i}}$ 为全岩样品的初始 $\delta^{18} \mathrm{O}$ 值, 初始值的计算结果见表 1 .

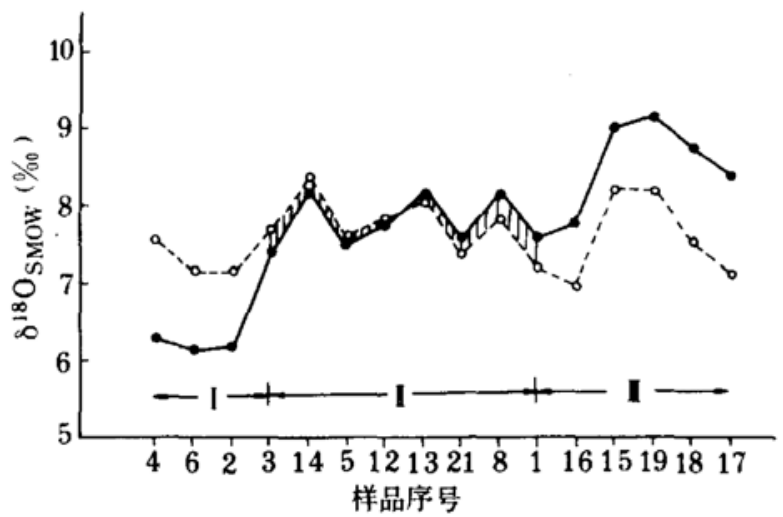

图 2 全岩氧同位素组成实测值与初始值的关系 - 为全岩测定值, $O$ 为全岩初始值

图 2 为表 1 中全岩样品氧同位素实测值 与其初始值的对应关系. 从中可以发现,如把 约 $0.16 \%$ 的分析误差考虑在内, 实测值与初 始值之间存在着三种关系: $\delta^{18} \mathrm{O}_{\mathrm{TR}}<\delta^{18} \mathrm{O}_{\mathrm{i}}$; $\delta^{18} \mathrm{O}_{\mathrm{TR}} \approx \delta^{18} \mathrm{O}_{\mathrm{i}} ; \delta^{18} \mathrm{O}_{\mathrm{TR}}>\delta^{18} \mathrm{O}_{\mathrm{i}}$. 因为成岩后 的低温蚀变或风化作用导致火山岩富集 ${ }^{18} \mathrm{O}$, 而高温蚀变却使其亏损 ${ }^{[3-5]}$, 因此从氧同位 素组成是否发生了变化的角度考虑, 陶波地 区的酸性火山岩可划分为三种类型: 高温蚀 变类型 (I 区域)、基本未蚀变类型 (II 区域) 和低温蚀变类型 (III 区域). 


\section{2 火山岩的成因}

众所周知, 火成岩的 $\delta^{18} \mathrm{O}_{i}$ 值, 主要受控于母岩浆和被其同化物质的氧同位素组成以及岩 浆结晶分异的程度 ${ }^{\text {》 }}$. 在陶波火山岩带之下或邻区, 已知有四类地质体: 上地幔、下地壳、 Torlesse 群和 Waipapa 群地层, 其平均 $\delta^{18} \mathrm{O}$ 值分别约为 5.8, 9.6, 12.0 和 $9.9 \%{ }^{[8,9}$. 研究表 明, 在火山岩浆温区内, 部分熔融产生的岩浆, 其 $\delta^{18} \mathrm{O}$ 值比源区物质约高 $0.5 \%$ 而绝不会低于 源区物质 ${ }^{[9]}$. 据表 1 , 陶波酸性火山岩的 $\delta^{18} \mathrm{O}_{\mathrm{i}}$ 值为 $6.78-8.47 \% 0$. 显然, 这些火山岩不太可能 单独由 Torlesse 群或 Waipapa 群地层或下地壳物质部分熔融而形成. 由于陶波酸性火山岩 的 $\delta^{18} \mathrm{O}_{\mathrm{i}}$ 与全岩 $\mathrm{SiO}_{2}$ 含量间不存在相关性 (图略), 且在 $10 \% \mathrm{SiO}_{2}$ 区间内, $\delta^{18} \mathrm{O}_{\mathrm{i}}$ 的变化已高达 约 2\%（表 1), 这表明结晶分异作用不可能是影响 $\delta^{18} \mathrm{O}_{\mathrm{i}}$ 的主要因素 ${ }^{[6,10,11]}$. 所以这些火山岩 亦不太可能仅由幔源岩浆的结晶分异而形成. 从氧同位素资料看, 它们最可能是幔源岩浆 $\left(\delta^{18} \mathrm{O} \approx 5.8 \%\right.$ ) 同化本区地壳物质 (下地壳、Torlesse 群、Waipapa群) 的结果.

\section{3 . 结 论 和 讨 论}

1. 陶波酸性火山岩中长石和石英斑晶在成岩后氧同位素组成基本无变化. 某些全岩样 品的氧同位素实测值偏离初始值, 是其基质在成岩后与流体发生同位素交换的结果. 因此, 不能把斑晶矿物或矿物对保持了其初始同位素组成特征,作为火山岩全岩也保持了其初始同 位素组成特征的判据.

2. 根据初始氧同位素组成, 陶波酸性火山岩应为地幔和地壳二端元混合的产物. 但是, 该区地壳端元较为复杂, Waipapa 群、Torlesse 群和下地壳这三部分地壳物质中, 究竟哪个 部分是构成酸性火山岩中地壳端元的主体, 氧同位素资料则难于作出定论. 这有待于进一 步工作.

\section{参考 文 献}

[1] Cole, J. W., N. Z. J. Geol. Geophys., 1979, 22:631-657.

[2] Tatsumi, Y., Tsunakawa, H., The Island Arc, 1992, 1:40-50.

[3] Taylor, Jr. H. P. et al., Earth Planet. Sci. Lett., 1984, 69:263-276.

[4] Ferrara, G. et al., Earth Planet. Sci. Lett., 1985, 75:13-38.

[5] Shieh, Y. N., Geochim. Cosmochim. Acta, 1985, 49:117-123.

[6] Blattner, P. et al., Earth Planet. Sci. Lett., 1983, 65:276-286.

[7] James, D. E., Ann. Rev. Earth Planet. Sci., 1981, 9:311-344.

[8] Graham, I. J. et al., Geochim. Cosmochim. Acta, 1992, 56:2797-2819.

[9] Blattner, P., Reid, F., Geochim. Cosmochim. Acta, 1982, 46: 1417-1429.

[10] Blattner, P. et al., Contrib. Mineral. Petrol., 1989, 101:438-446.

[11] Hu, R. Z. et al., Chinese Sci. Bull., 1992, 37:317-320. 